\title{
Technologiegestützter After Sales Service
}

\author{
Margit Scholl
}

\section{Motivation}

Die unternehmensweite Integration von Daten, Prozessen und Dienstleistungen wird auch für KKU (Klein- und Kleinstunternehmen) immer notwendiger und verlangt die Konzeption von unterstützenden Anwendungssystemen, die für das hier betrachtete Klientel in einfacher Art und Weise handhabbar sein müssen. Gleichzeitig wird eine aktuell gehaltene Qualifikation der Fach- und Führungskräfte angesichts des fortschreitenden Strukturwandels hin zur wissensbasierten Informations- und Dienstleistungsgesellschaft zum entscheidenden Standortfaktor, von dem die Innovationskraft und Wettbewerbsfähigkeit der Wirtschaft abhängt. In diesem Beitrag soll ein praxisorientierter Lösungsansatz aufgezeigt werden, um Produkte und Dienstleistungen zwischen KMU bzw. zwischen KMU und öffentlicher Verwaltung zu vermitteln, transparent darzustellen und damit letztlich Kundenzufriedenheit und Kundenbindung zu optimieren. Gemeinsam mit Partnern wurde die Idee entwickelt und praktisch umgesetzt, dass die Optimierung von Prozessen durch Nutzung einer Lernplattform via Internet möglich ist, bei gleichzeitiger Senkung der Prozesskosten durch webbasierte Qualifizierung und Betreuung. Die bisherigen Ergebnisse dieses Entwicklungsprozesses wurden im September 2006 auf dem 3. Fernausbildungskongress an der Bundeswehrhochschule in Hamburg im Workshop „Möglichkeiten technologiegestützter Bildungskonzeptionen für KMU“ als Erfahrungsworkshop präsentiert [1].

\section{Hintergrund}

Über den vielfältigen Einsatz einer extrem einfachen und intuitiv nutzbaren eLearning-Plattform konnte bereits an dieser Stelle berichtet werden [2]. Die Anwendungsberei-

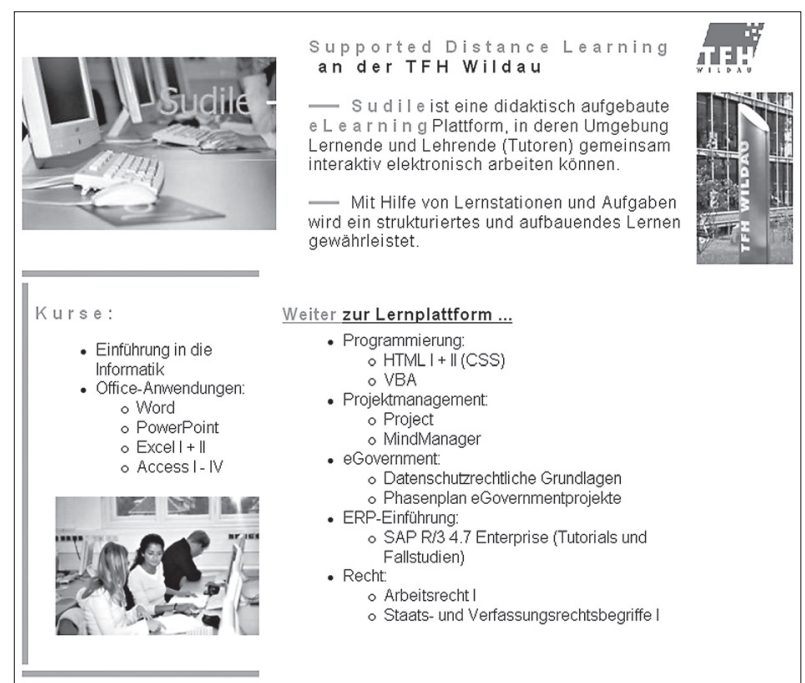

Abb. 1: Zugangsseite zur Sudile-Lernplattform des Fachbereichs Wirtschaft, Verwaltung und Recht, http://www.sudile.com/tfh-wildau che für eLearning-Kurse werden unseren Möglichkeiten entsprechend kontinuierlich erweitert wie es die Abb. 1 verdeutlicht.

Da barrierefreie Anwendungen inzwischen ein unverzichtbares Qualitätsmerkmal moderner Software sind, das auch vom Gesetzgeber eingefordert wird, wurde die Lernplattform im letzten Jahr barrierefrei umgestaltet. Der Umgestaltungsprozess, die Einhaltung allgemeiner Standards und aufgetretene Probleme konnten im Februar 2006 auf dem Internationalen Rechtsinformatik Symposion in Wien aufgezeigt und erläutert werden [3]. Im Mai diesen Jahres konnten wir die Plattform und Kurse auf der ersten internationalen eLearning-Konferenz Afrika in Addis Abeba präsentieren [4].

\section{Das neue Anwendungsfeld}

Ziel eines internen, kooperativen Forschungsprojektes der Autorin war es, beispielhaft für das Hauptgeschäftsfeld „Reinigung" der bebra-Gesellschaft für Verwaltungsentwicklung $\mathrm{mbH}$, die darin enthaltenen Verfahrenshinweise für die Kunden (Kommunen) in einer didaktisch fundierten und elektronischen Form mit Hilfe der Lernplattform der Sudile-durchdachtes eLernen GbR so aufzubauen und bereit zu stellen, dass sie zukünftig als integraler Bestandteil der Geschäftsprozesse in die Dienstleistung der bebra $\mathrm{GmbH}$ eingehen können. D.h., Ausgangspunkt unserer Idee der Prozessoptimierung sind interne Behördenprojekte, die unter Hinzuziehung von Dienstleistern u. a. Kostensenkungen zum Gegenstand haben. Über Kostenreduzierung lassen sich interne Behördenprojekte finanzieren. Mit dem Umsetzen von Prozesswissen in das Lernsystem resultiert ein zeit- und ortsunabhängiger, aktiver und nachhaltiger Erwerb von professionellem, angewandten Wissen für alle beteiligten Verwaltungsmitarbeiter (als Kunden) bei gleichzeitiger Optimierung der Betreuung durch die beratende Firma (als Dienstleister).

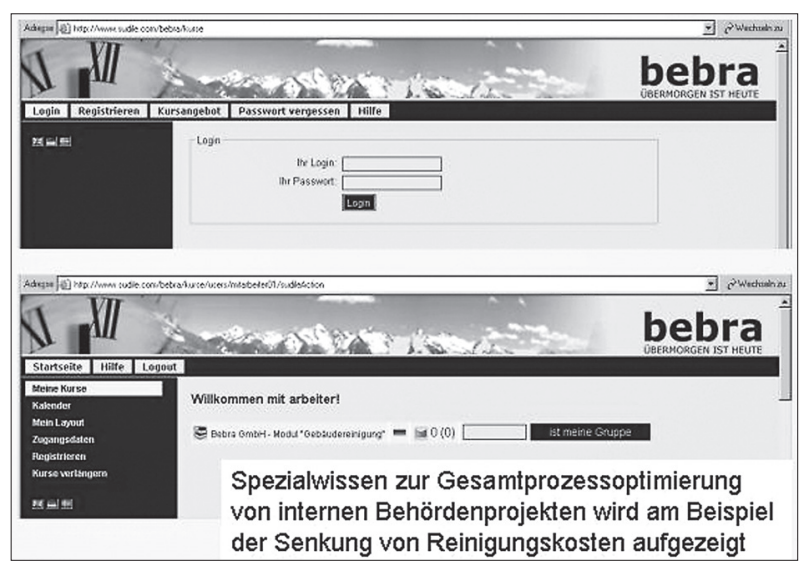

Abb. 2: Layoutanpassung der Plattform an das $\mathrm{Cl}$ der bebraGesellschaft für Verwaltungsentwicklung $\mathrm{mbH}$ 
Als konkretes Beispiel wurde in partnerschaftlicher Kooperation das Prozesswissen zur Senkung von Reinigungskosten in die Lernplattform umgesetzt. Dabei musste das Aussehen des Lernsystems dem CI (Corporate Identity) der bebra $\mathrm{GmbH}$ angepasst werden (s. Abb. 2).

Erste Ergebnisse konnten am Stand der bebra $\mathrm{GmbH}$ auf der Messe „Moderner Staat“ im November 2005 in Berlin gezeigt werden. Das für die Geschäftsprozesse notwendige Spezialwissen wurde unter pädagogischen und didaktischen Gesichtspunkten als elektronische Lerneinheiten praxisorientiert an der konkreten Dienstleistung vom Geschäftspartner selbst eingepflegt und kann jetzt als tägliche Businesshilfe genutzt werden (s. Abb. 3). Von Bedeutung sind dabei die extrem einfache, intuitive Handhabung für alle beteiligten Nutzergruppen und die vielfältigen Kommunikationsformen wie Chat, Wiki, Forum und E-Mail. Das zu vermittelnde Wissen in Firmen kann sehr umfangreich sein und die Zeitknappheit der Firmenmitarbeiter für die Inhaltserstellung ist ein zentrales Problem. Umso wichtiger ist die Einfachheit der Inhaltserstellung und ein schneller, unkomplizierter Umgang mit dem Anwendungssystem.

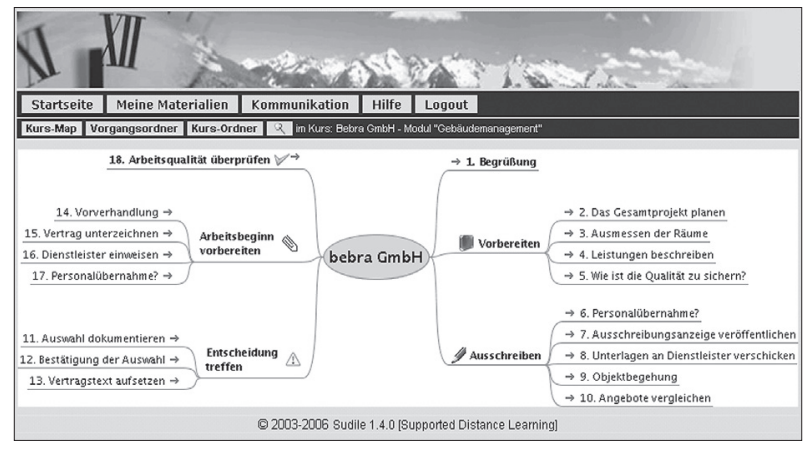

Abb. 3: Prozesswissen der Dienstleistung umgesetzt in einen eLearning-Kurs. Übersicht des Kurses "Gebäudereinigung" als Mindmap für die im Prozess involvierten Mitarbeiter. Das Design der Plattform verweist darüber hinaus auf die weiteren Kommunikations- und Arbeitsmöglichkeiten.

Über das Vorgehen zum Aufbau des Kurses „Gebäudereinigung“, den aktuellen Stand und die konkreten Erfahrungen konnte auf dem Internationales Rechtsinformatik Symposium 2006 im Workshop „Wissensbasiertes Prozessmanagement in Verwaltungsnetzwerken“ berichtet werden [5]. Die Umsetzung im Kooperationsprojekt erfolgte in drei Phasen:

- Strukturierung der Prozessinformationen

- Erstellung der spezifischen Inhalte

- didaktische Überarbeitung.

Eine der wichtigsten Erkenntnisse für den Dienstleister bebra GmbH war, dass die Systematisierung und Überarbeitung der eigenen Prozesse zur Kundenbetreuung nicht nur für die Verwaltung (den Kunden), sondern auch für den Dienstleister selbst einen Gewinn darstellen. Während der aktuellen Projektarbeit entwickelte sich das Lernsystem zur Hilfe für konkrete Wissensaneignung und als Nachschlagewerk. Die vielfältigen Kommunikationsformen und die Dokumentenstandardisierung führt zu einer gezielteren Projektbetreuung, erhöhten Lernbereitschaft durch Qualifizierung während des Projektes und am Ende zur Qualitätssicherung durch Übergabe von standardisierten Leistungen.
Zusammenfassend halten wir die folgenden Aspekte unseres Ansatzes innovativ:

- Relevante Inhalte werden vom Geschäftspartner in einfacher Weise selbst erstellt

- Inhalte sind praxisorientiert am tatsächlichen Businessprozess angepasst

- Geschäftsprozesse werden für alle Beteiligten systematisiert und transparenter

- Notwendiges Wissen führt zu einer zielgerichteten Qualifizierung aller Beteiligten.

Wir halten unseren Ansatz generell auf viele Dienstleistungsprozesse zwischen Geschäftspartnern anwendbar. Insbesondere wird er auch interessant für Abläufe mit geringer Durchlaufquote und hoher Vergessensrate, da das Anwendungssystem dann als Nachschlagewerk fungieren kann. Einmal übertragen, werden auch die Einsparpotenziale unseres Ansatzes sichtbar: Vorbereitungs-, Reise-, Beratungszeit werden minimiert und weniger Präsenz beim Kunden ist notwendig. Die standardisierte, aber flexible gemeinsame Arbeitsgrundlage der Geschäftsprozesse vermeidet Missverständnisse und spart ebenfalls Zeit. Demgegenüber werden die Kommunikationsmöglichkeiten webbasiert verbreitert und qualifizieren die Beteiligten ebenfalls.

\section{Ausblick}

Der dargestellte Ansatz scheint auch dafür geeignet, dass sich KKU über dieses produktionsbezogene Nachdenken und Darstellen ihrer Dienstleistung in einem Lernsystem im Wettbewerb besser behaupten können. Denn diese Entwicklung hat zur Folge, dass die Mitarbeiter automatisch stärker in eine lebensbegleitende Weiterbildung einbezogen werden. Nach wie vor besteht ein enormes Potenzial an betrieblichen Einsatzmöglichkeiten von eLearning via Internet. Doch Untersuchungen zeigen [6], dass der Umfang der Nutzung von eLearning insbesondere für KMU weit hinter den Erwartungen zurückbleibt. Gerade kleine und mittlere Unternehmen sind es aber, die von den organisatorischen Vorteilen, die eLearning bietet, besonders profitieren könnten. Denn gerade sie sind darauf angewiesen, Weiterbildung flexibel und arbeitsplatznah ohne längere Freistellungen zu organisieren. Ein kostengünstiges eLearning für passgenaue Qualifizierung ist für KKU dringend notwendig! Doch wie kann man es sinnvoll nahe bringen? Zumal ein elektronischer Weiterbildungsmarkt existiert, der nicht auf die Bedürfnisse von KMU zugeschnitten ist. In einer Studie des europäischen Kooperationsprojekts „Ariel“ (Analysing and Reporting on the Implementation of Electronic Learning in Europe, [7]) wird zusammengefasst, dass KMUs von Entwicklern einerseits „als Zielgruppe verschmäht" werden und andererseits Produkte nicht geeignet sind und am Bedarf der Zielgruppe vorbei gehen. Unsere Idee ist, elektronische Fort- und Weiterbildung anhand der elektronischen Optimierung von Geschäftsprozessen im Bereich der KKU zu etablieren. Mit den eigenen Geschäftsprozessen als Basis dürften die KKU dem Thema offener gegenüber stehen.

Daher haben wir im BMBF-Programm zur Förderung angewandter Forschung an Fachhochschulen im 
Verbund mit der Wirtschaft $\left(\mathrm{FH}^{3}\right)$ den Projektantrag „Blended Coaching als Basis für ein integratives Dienstleistungskonzept für KKU“ eingereicht. Darin wird eine webbasierte Qualifizierung und Betreuung vorgestellt, die als internetgestütztes Coachen bezeichnet werden kann - wir nennen es „Blended Coaching“. Es stellt eine neue Form der Kundenbetreuung innerhalb der Dienstleistungen dar, die einerseits eine höhere Transparenz der Arbeitsprozesse ermöglicht und anderseits den Kunden höhere Eigenverantwortung abverlangt. Neben dem individuellen Know-how-Gewinn und der Förderung eines lebensbegleitenden Lernens im betrieblichen Umfeld, das konkret in die jeweiligen Geschäftsprozesse integriert werden soll, wird das Projekt die Stabilität von Unternehmensstrukturen der beteiligten KKU deutlich stärken. Es bleibt zu hoffen, dass die AiF-Begutachtung positiv ausfallen und zu einer Förderung führen wird.

\section{Literatur}

[1] Scholl, M., T. Dressler, Ch. Niemczik und El. Brenstein; eBusiness + eGovernment + eLearning: Technologiegestützter After Sales Services in KMU, Workshop, 3rd Distance Training Convention, 19.-21.9.2006, Helmut-Schmidt-Universität, Universität der Bundeswehr, Hamburg, 2006.

[2] Scholl, M.; Einführung von eLearning am Fachbereich Wirtschaft, Verwaltung und Recht - Konsequenzen und Ausblick, TFH Wildau, Wissenschaftliche Beiträge, Heft 2005.

[3] Koppatz, P., Ch. Niemczik und M. Scholl; Online für alle: Barrierefreier Zugang zu Webinhalten;IRIS 2006, Internationales Rechtsinformatik Symposium, Wien, 16.-18.2.2006; Schriftenreihe Rechtsinformatik, Druck im Erscheinen, 2006.

[4] Scholl, M. und Ch. Niemczik; SUDILE learning platform - Blended Learning Scenarios at the University of Applied Sciences Wildau (Demonstration); 1st International Conference on ICT for Development, Education and Training; ELearning Africa! UNCC, Addis Ababa, Ethiopia, May 24 - 26, 2006, forthcoming. (2006)

[5] Scholl, M., H. Jung und Ch. Niemczik; Qualifizierung und Einsparungen in den Verwaltungen - durch webbasierte Betreuung zum Erfolg, Workshop „Wissensbasiertes Prozessmanagement in Verwaltungsnetzwerken“, IRIS 2006, Internationales Rechtsinformatik Symposium, Wien, 16.-18.2.2006, Schriftenreihe Rechtsinformatik, Druck im Erscheinen. (2006)

[6] Loebe und Servering (Hrsg.), eLearning für die betriebliche Praxis, f-bb gGmbH, Bertelsmann Verlag, Bielefeld 2003, ISBN 3-7639-3112-0.

[7] http://www.hyblearn.odl.org/EN/Home.htm, http://www. checkpoint-elearning.de/article/1053.html

\section{Autorin}

Prof. Dr. Margit Scholl

Technischer Fachhochschule Wildau

Fachbereich Wirtschaft, Verwaltung und Recht

Tel. +49 1723214682

margit.scholl@tfh-wildau.de

http://www.tfh-wildau.de/scholl

\section{Partner}

Horst Jung, Tobias Dressler

bebra-Gesellschaft für Verwaltungsentwicklung mbH

Veilchenweg 5 D , 14772 Brandenburg

Christian Niemczik

Sudile - durchdachtes eLernen GbR

Jägerstraße 36 , 14467 Potsdam

\author{
Dr. Elke Brenstein \\ Lernen-Gestalten \\ In der Heide 4, 14476 Potsdam
}

